\title{
On the lateral dominance in locus exploration human motor space
}

\author{
TAKASHI FUJIOKA ${ }^{1}$
}

Department of Educational Psychology, Faculty of Education, Kyushu University ${ }^{2}$

\begin{abstract}
The purpose of this study is to examine the lateral dominance in the learning of locus exploration in human motor space. Standing blindfolded subjects were asked to explore a locus of space by using a pen. Four $(2 \times 2)$ conditions were set, combining the hand to be performed (left and right), and availability of visual feedback (visual feedback and non-visual feedback) they will be designated as: VR, VL, NVR, and NVL. Fifteen trials were combined to four blocks. The chief findings were as follows: 1) At block 1, left hand was more accurate than right hand, while in the later block right hand was more accurate. 2) For right hand, greater consistency in locus exploration was observed in the visual feedback condition than in the non-visual feedback one. 3) For the non-visual feedback condition, greater consistency was observed in left hand than in right hand. These findings suggest that lateral dominance in hand operation was attributed to the different structure in human motor space. We inferred that there is a lateral dominance in the subject's set which divides human motor space into external or internal space.
\end{abstract}

Key words: human motor space, lateral dominance, motor learning, external space, internal space.

Some of recent researches on laterality demonstrated that right hand is more dominant in such tasks as tapping, drawing, gripping while, left hand is more dominant in such tasks as finger spreading, finger posturing (Kimura \& Vanderwolf, 1970; Ingram, 1975; Naruse, 1979). Such findings may be explained in terms of structural different human motor space. Naruse (1973) inferred that human motor space is composed of two components; body space as one's own body and physical and geometric space as an object. When the former is assumed to be internal space and the latter to be external space, respectively, a subject seems to make contact with external space through internal space, especially using two hands for upper part of space. Reviewing the previous studies

1 The author thanks Professor Gosaku Naruse of Kyushu University (now at Kyushu Woman's University), for his critical reading and helpful suggestions on this manuscript.

2 Now at the Department of Psychology, Faculty of Education, Yamagata University, Koshirakawacho, Yamagata 990.
(Ingram, 1975; Naruse, 1979) on human motor space, right hand seems to be dominant for tasks in the objective world or external space, while left hand in tasks for one's own body or internal space.

Then, it is inferred that for right-handed subjects right hand is more dominant in object operation tasks over left hand, while, left hand is more dominant in selfbody operation tasks over the right hand. Therefore, with right hand the subject would utilize the external or visual information, while, with left hand the subject would utilize external information very little. External information would be utilized very well for the subject who accepts tasks in external space, while internal or proprioceptive information rather than external information would depend on for the subject who accepts tasks in internal space. From this standpoint, the present experiment was designed to examine how the subject uses the visual feedback as external information through each hand. There are few experimental studies which investigated this important problem. 
For this purpose, we used a motor learning task, specifically an exploratory task of spatial location. According to Connolly (1977), motor learning has been considered as the relation between mental activities and motor behavior. This task would be switched with the object operating task or self-body operating task according to a subject's mental activities, especially his or her choice of external space or internal space. Therefore, it would be examined by this motor learning task whether or not the process of motor behavior through each hand is affected by visual feedback as the external information with relation to this choice.

\section{Method}

\section{Subjects}

Subjects were right-handed male $(n=$ 16) and female $(n=16)$ university students who volunteered to participate in the study. Handedness was evaluated by means of H. N. Handedness Inventory (Hatta \& Nakatsuka, 1975). No subject had prior experience with the learning task.

\section{Apparatus}

A board $(60 \times 42 \mathrm{~cm})$ was used in this study. At the center of this board a hole of $3 \mathrm{~mm}$ in diameter as a target was bored to a depth of $2 \mathrm{~mm}$. Subjects were instructed to slant a line by using a ceramic ball-point pen from the starting point to the target. Only one starting point was set for each hand. The starting point on the board was $30 \mathrm{~cm}$ away from the target, at an angle of $30^{\circ}$ from the vertical line which contained the target, and on the right side of the target for right hand and on the left side for left hand.

\section{Procedure}

Each blindfolded subject was led to a standing position in front of the board on the wall. The subject had been blindfolded before entering the testing room so the subject could not see the apparatus and his physical relation to it. The distance from the board was estimated to be three quarters of the length of the subject's arm. The target was located at the same height as the upper edge of the subjects' chest bone and $10 \mathrm{~cm}$ to the right from the subject's midline for right hand, $10 \mathrm{~cm}$ to the left for left hand. The subject, however, was not aware that the target was in that position.

The subjects were instructed to explore the target on the board by using the pen. Each subject was familiarized with the target area by moving his hand around the perimeter of a square $(30 \mathrm{~cm}$ aside) which contained the target. This was performed with the assistance of the experimenter. The experimenter guided the subjects' hands to the starting point. Then, the subjects moved their hands directly to the target, and when they weren't able to hit the target, they were allowed to change the direction of pen movement toward the target up to two times. Prior to the experimental trials, the subject practiced moving the pen on the white paper at a speed of $7 \mathrm{~cm}$ per second and continued these trials until they could move the pen within an error of $1 \mathrm{~cm}$. The Digital Timer measured this speed from the start point to end point of the line $(7 \mathrm{~cm})$ on the white paper.

The four conditions were defined by the hand used for moving the pen and the source of feedback available for motor control. Specifically, they were (a) righthand control with non-visual feedback (NVR), (b) left-hand control with nonvisual feedback (NVL), (c) right-hand control with visual feedback (VR), and (d) left-hand control with visual feedback (VL).

NVR, NVL groups. The subjects were informed that after moving their pens in a straight line toward the target three times and not hitting it, they were then allowed to move their pens freely until they reached it. 
$V R, V L$ graups. The procedures for VR, VL groups followed those for NVR, NVL groups during initial five trials. After six trials, the subjects were allowed to take off their eye-masks and were able to use visual feedback; that is, after three linear movements, they could ascertain the location of the pen, the target and motion of the pen on the board to the target by following the light. The light regulated the visible range with a radius of $10 \mathrm{~cm}$ round the target as a center, so the subject could not see the starting position and the locus from that position. After two seconds, it was extinguished. The testing room was entirely dark before the light was switched on.

Four independent groups ( $n=8$ in each) received 15 trials divided into four blocks (block 1, 2-4; block 2, 7-9; block 3, 10-12; block 4, 13-15). Initial five trials for the subjects were performed under the same conditions.

Localization errors of one, two or three points made by the locus of three linear movements (the terminal points of each linear movement) deviated from the point of the target were recorded to the nearest millimeter, and absolute error (AE), constant error (CEx, CEy), and variable error (VEx, VEy) were calculated. AE is the absolute deviation of the exploratory point from the criterion target and represents an estimate of general movement accuracy. $\mathrm{CE}$ is the signed (+ or - ) deviation of the exploratory point from the criterion target and is regarded as an index of movement bias: CEx is the degree of undershooting revealing the deviation of the right direction from the target for right hand, the one of the left direction for left hand around the criterion target and overshooting revealing the deviation of the left direction from the target for right hand, the one of the right direction for left hand around the criterion target; CEy is the degree of uppershooting revealing the deviation of the upper direction from the target for each hand around the criterion target and lower shooting revealing the deviation of the lower direction from the target for each hand around the criterion target. VEx and VEy are standard deviations of the signed scores around their CEx and CEy mean, and are an estimate of variability or consistency of exploratory movement.

Data from all the trials were in a $2 x$ $2 \times 4$ (Feedback conditions $\times$ Hands performed $\times$ Blocks) factorial analysis of variance (ANOVA) with repeated measures on the third factor.

\section{Results}

The $\mathrm{AE}$ analysis indicated that the block main effect $[F(3.84)=24.61, p<.001]$ and the hand block interaction $[F(3.84)=$ $4.65, p<.01]$ were significant, and the hand $\times$ feedback condition block interaction $[F(3.84)=2.47, p<.10]$ were not significant (Fig. 1). A post hoc analysis of the interaction was carried out (using

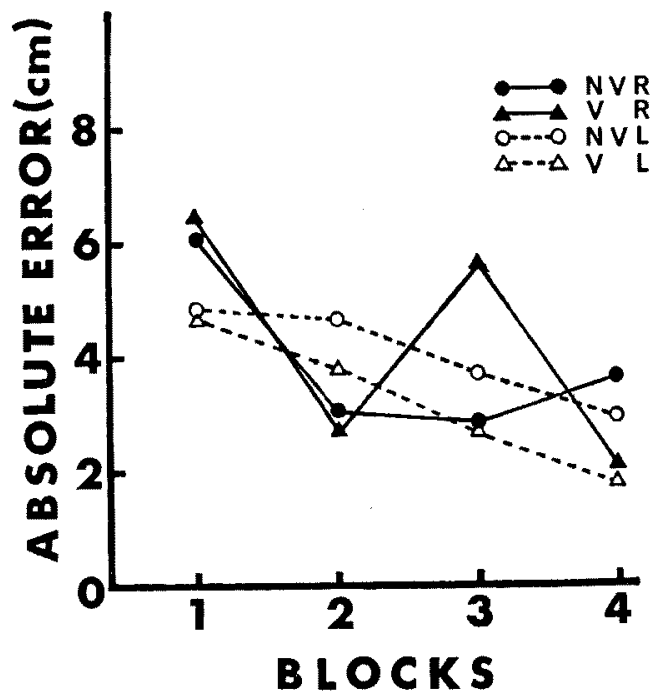

Fig. 1. Mean Absolute Error of left and right hands in the visual and non-visual feedback conditions as a function of four blocks (NVR, right hand control with non-visual feedback; VR, right hand control with visual feedback; NVL, left hand control with non-visual feedback; VL, left hand control with visual feedback). 
Tukey's HDS procedure). This analysis showed that, at right hand block 1 was less accurate than the other blocks; 1 (mean absolute error of the sum of $\mathrm{V}$ condition with NV condition, $\bar{x}=6.36 \mathrm{~cm})-2$ $(\bar{x}=2.98)[q(2.83)=8.6, p<.01], 1-3(\bar{x}=$ 4.27) $[q(4.84)=9.12, \quad p<.01], \quad 1-4 \quad(\bar{x}=$ 2.94) $[q(3.84)=8.9, p<.01]$, while, at left hand block $1(\bar{x}=4.89)$ was less accurate than block $3(\bar{x}=3.28)$ and block $4(\bar{x}=$ 2.39) $[q(3.84)=4.41, p<.01 ; \mathrm{q}(4,84)=$ $6.92, p<.01],[q(3.84)=5.29, p<.01]$.

At block 1, right and left hands were as accurate in the visual feedback condition (mean absolute error, right, $\bar{x}=6.51$; left, $\bar{x}=4.81$ ) as in the non-visual feedback one (right, $\bar{x}=6.21$; left, $\bar{x}=4.98) \quad[q(2,112)=$ $0.24, p>.05][q(2,112)=0.35, p>.05]$.

At block 1 , left hand $(\bar{x}=4.89)$ was more accurate than right hand $(\bar{x}=6.36)[q$ $(2,112)=2.97, p<.05]$, but at block 2 the opposite result was observed (right, $\bar{x}=$ 2.98; left, $\bar{x}=4.32)[q(2,112)=3.22, p<$ $.05]$. And at block 3 , only in right hand the visual condition $(\bar{x}=5.82)$ was less accurate than the non-visual condition $(\bar{x}=$ $3.01)[q(2,112)=3.21, p<.05]$.

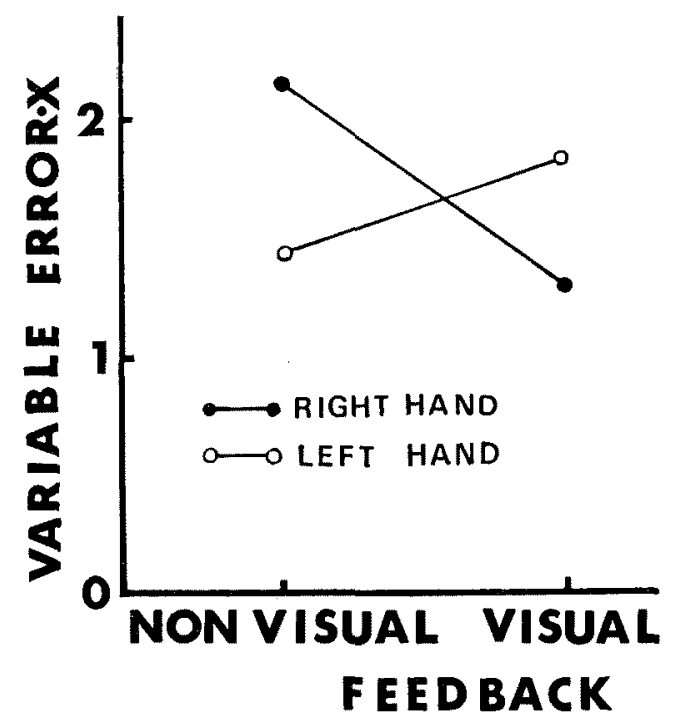

Fig. 2. Mean Variable Error (x) of left and right hands as a function of two feedback conditions.
The $\mathrm{CEx}$ and $\mathrm{CEy}$ analysis indicated that all the main effects and interaction were not significant.

The VEx analysis of block 2, block 3 and block 4 demonstrated that the feedback condition $\times$ hand interaction was significant $[F(1,28)=4.88, p<.05]$. Subsequent analysis showed that, at right hand greater consistency was observed in the visual feedback condition (mean variable error, $\bar{x}=1.32$ ) than in the non-visual feedback one $(\bar{x}=2.13) \quad[F(1,28)=4.65, p<$ $.05]$, while at the non-visual feedback condition greater consistency was observed in left hand $(\bar{x}=1.43)$ than in right hand $(\bar{x}=2.13)[F(1,28)=3.50, p<.05]$ (Fig. 2). The VEy analysis indicated the same results as the VEx analysis by the feedback condition $\times$ hand interaction $[F(1,28)=$ $3.28, p<.10]$. The hand main effect was significant $[F(1,28)=7.48, p<.01]$, and greater consistency was observed in left hand (the non-visual feedback condition, $\bar{x}=1.20$; the visual feedback condition, $\bar{x}=$ 1.48) than in right hand (the non-visual feedback condition, $\bar{x}=2.46$; the non-visual feedback condition, $\bar{x}=1.72$ ).

\section{Discussion}

The purpose of this study was to examine how each hand utilizes external information or visual feedback. It was found that for right hand the subjects can correct the initial errors by operating the target or the external object. However, at 3 block, the subjects increased errors for right hand especially under visual feedback condition. It would be inferred that visual feedback disturbed their proprioceptive sensation. From these findings, it was predicted that the subjects would usually tend to relate his right hand to the external space actively and widely. For left hand, however, the subject did not make more errors in the initial trials than for right hand, and were able to correct the errors gradually.

Accordingly, for left hand, the subjects 
would regard this task as a self-body task, and utilize their proprioceptive sensation from the initial trials. Therefore, at block 1 , the subjects were able to perform more accurately with left hand than with right hand in spite of the unfamiliar blindfolded situation. It was found that for right hand they were able to perform the tasks freely by using external information such as visual feedback, while left hand was not affected by external information.

Therefore, the present study ascertained that right hand is dominant in object operation tasks, or has human motor space opened to external space, while left hand is dominant in the self-operation tasks or has human motor space closed in internal space.

Moreover, a subject of NVL group reported after this experiment: "I thought that left hand is a useless hand and I supported the pen rather than grasping it, and I wanted to find the target." On the other hand, a subject of NVR group reported: "I aimed at the target, and then attacked it." These reports supported the findings of this experiment in a more concrete way. The subject would have different set using each hand; the mental set to external world focused on the target for right hand, and the another mental set for left hand to internal world focused on his or her body.

The past motor learning research (Woodworth, 1896) described right hand as more skillful than left hand. From this experiment, however, it was suggested that left hand is dominant to right hand in the initial trials, and in addition, there were some differences in performance, learning process, and the way of utilizing the external information by means of the subject's choice of the external space or internal space for each hand.

Much research on laterality (for example, Downey, 1933; Harris, 1958) has been undertaken from the accepted view-point that emphasized only the performance of each hand. However, this experiment demonstrates the difference in the subjects' set to external or internal space for each hand, as well as the difference in performance shown in the more recent experiments.

In conclusion, we operate right hand as if "there" is a target as an object, and left hand as if "here" is a target as a part of human body. We assume that lateral dominance in both hands was supported by these differences in operation of human motor space. It is hoped that future analysis will reveal the integration of mental set to external and internal world for coordinating each hand such as playing piano or professional skills.

\section{References}

Connolly, K. 1977 The nature of motor skill development. Journal of Human Motor Studies, 3, 128-143.

Downey, J. E. 1933 Laterality of function. Psychological Bulletin, 30, 109-142.

Harris, A. J. 1958 Harris tests of lateral dominance, manual of directions for administration and interpretation. 3rd ed., New York: Psychological Corporation.

Hatta, T., \& Nakatsuka, Z. 1975 H. N. Handedness inventory. In S. Ono (Ed.), Paper in celebration of 63rd birthday of Prof. $K$. Ohnishi. Osaka City University, Pp. 224-247. (In Japanese)

Ingram, D. 1975 Motor asymmeries in young children. Neuropsychologia, 13, 95-102.

Kimura, D., \& Vanderwolf, C. H. 1970 The relation between hand preference and the performance of individual finger movements by left and right hands. Brain, 93, 769-774.

Naruse, G. 1973 Psychological rehabilitation. Seishin Shobou. (In Japanese)

Naruse, G. 1979 A comparative study of lateral dominancy in finger-hand works. Research Bulletin, Educational Psychology Section, the Faculy of Education, Kyushu University. Vol. 24. No 2. 111. (In Japanese with English summary)

Woodworth, R.S. 1896 The accuracy of voluntary movement. Psychological Review Monograph Supplements, 3, No. 3.

(Received June 30, 1986; accepted Sept. 10, 1988) 